Nuclear Physics B265 [FS15] (1986) 161-186

(C) North-Holland Publishing Company

\title{
THREE-DIMENSIONAL SPIN SYSTEMS WITHOUT LONG-RANGE ORDER
}

\author{
Francisco C. ALCARAZ \\ Departamento de Física, Universidade Federal de São Carlos, 13560 São Carlos, SP, Brasil \\ Laurence JACOBS \\ AT\&T Bell Laboratones, Holmdel, NJ 07733 USA \\ and \\ Institute for Theoretical Physics, University of Callfornia, Santa Barbara, CA 93106, USA
}

Robert SAVIT ${ }^{2}$

Physics Department, Universtty of Michtgan, Ann Arbor, MI 48109, USA

Received 15 April 1985

\begin{abstract}
Several examples of three-dimensional statistical models with multibody interactions and unusual symmetry properties are studied from a unified point of view. The symmetries of these theories are neither global nor local, but are something in between In the nomenclature of a recently proposed classification scheme, these theories have a symmetry index, $n \leqslant 2$, and so have no long-range order when the internal symmetry is continuous Duality properties and topological excitations of the theories are studied, and their phase diagrams are qualitatively discussed. Similarities between these three-dimensional semi-local theories and ordinary globally-symmetric theories in one and two dimensions are discussed.
\end{abstract}

\section{Introduction}

Recently, a scheme for the classification of statistical systems possessing multibody interactions whose symmetries are more general than the usual global or local symmetries has been proposed [1] and has led to the identification of a new symmetry index, $n$, for these theories. Just as the usual globally-symmetric statistical theories with continuous internal symmetries have a lower critical dimension, $d=2$, systems with more general symmetries have a lower critical value for their associated symmetry index. A theorem was recently proved [2] which shows that, regardless of the dimensionality of a system, theories with a U(1) symmetry with $n \leqslant 2$ have no long-range order. Apart from the intrinsic theoretical interest embodied in these systems, statistical models with $n=2$ (and other closely related models) are thought to be relevant to the description of certain aspects of liquid crystals and helical magnets. This paper is concerned with a fuller discussion of the marginal case;

1 Work supported in part by Conselho Nacional de Desenvolvimiento Cientıfico (Brazil).

2 A.P Sloan Foundation Fellow Research supported in part by the National Science Foundation under Grant PH78-0846. 
$n=2$. We will make a number of general observations about $n=2$ theories and study in some detail several $n=2$ statistical theories in three dimensions. As suggested by the theorem of ref. [2], we shall find that these models have critical properties qualitatively similar to ordinary globally-symmetric statistical models in two dimensions. It is our intention that the study of these models at their lower critical value of $n$ be a first step in the systematic study of the critical properties of the rich set of theories included in our classification scheme.

In subsect. 2.1 we will define what is meant by an index- $n$ symmetry in its simplest sense. In subsect. 2.2 we will describe the proof of the theorem which states that theories with a $U(1)$ symmetry and $n \leqslant 2$ have no long-range order. In subsect. 2.3 we will discuss an extension of the definition of index $-n$ symmetries to a wider class of theories, and we will argue that the theorem of subsect. 2.2 applies to this larger class as well. Subsect. 2.4 contains some additional, miscellaneous comments. In sect. 3 we will analyze three closely related three-dimensional models, all of which have an $n=2$ symmetry in the restricted sense of subsect. 2.2 but which differ in detail. These models are quite simple and serve to clarify most of the general features of $n=2$ symmetries. In particular, we shall discuss their properties under duality transformations and the relations between order and disorder variables. Next we present the results of Monte Carlo simulations of the $Z_{N}$ version of one of these models. These simulations lend strong support to the general theoretical expectations for this class of systems; in particular, they verify the prediction that the phase diagrams of $n=2$ theories with a $Z_{N}$ symmetry are qualitatively quite similar to those of the $d=2$ globally-symmetric, two-dimensional clock models [3]. Lastly, we shall briefly describe two somewhat more complex $n=2$ theories which have been discussed in the literature [4-6] and which, in a sense, motivated much of the present work. These two theories are examples of the generalized $n=2$ theories discussed in ref. [1].

We summarize our results in sect. 4 and conclude with some suggestions for further work. Finally, we show how to construct the hamiltonian formulation of one of our models in an appendix.

\section{Preliminaries}

\subsection{INDEX- $n$ SYMMETRIES}

The theories we will discuss in this paper will be statistical theories of a single field which is associated with the sites of a lattice. In this section we shall consider those theories which have a $U(1)$ internal symmetry with the site-associated spins given by $s(\boldsymbol{r})=\mathrm{e}^{i \phi(r)}$. For simplicity we will consider theories defined on a $d$ dimensional simple hypercubic lattice. The defining characteristic of our models is that they are described by hamiltonians which are invariant under the transformation

$$
\phi\left(x_{1}, \ldots, x_{d}\right) \rightarrow \phi\left(x_{1}, \ldots, x_{d}\right)+\Lambda\left(x_{n+1}, \ldots, x_{d}\right) .
$$


These theories will be said to possess a symmetry index equal to $n$. (Actually, most of the results of this paper apply to a wider class of theories. This generalization is defined in subsect. 2.3.) In fact, most of the models we will encounter have a somewhat more complex symmetry structure in which there may be more than one distinct semi-local symmetry (see, e.g., the theory defined in eq. (3.4) below). Such theories will be said to have a compound symmetry.

As we have discussed elsewhere [1], the hamiltonians of our models can be written in the form

$$
H(\phi)=\sum_{x} \sum_{p=1}^{s} f_{p}\left(\sum_{j=1}^{q(p)} c_{p j} \phi_{j}\right),
$$

where the $f_{p}$ are functions of a linear combination of $q(p) \phi$ 's and the $c_{p j}$ are some set of constant coefficients. The coordinate of $\phi_{J}$ is $x+r_{j}$. We will assume that there are no explicit long-range interactions, so that all $\left|\boldsymbol{r}_{j}\right|$ are finite. For the sake of simplicity, we shall focus our attention on theories for which the interactions in (2.2) are defined on the $k$-dimensional $(k \leqslant d)$ simplices of the $d$-dimensional lattice. For example, nearest-neighbor interactions along links, four-body interactions around a plaquette, etc. Our results apply as well in more general cases. (See ref. [1] and the examples below.)

\section{ABSENCE OF LONG-RANGE ORDER FOR $n \leqslant 2$}

It was recently shown [2] that theories with $n=1$ or $n=2$ symmetry indices do not have long-range order for any $T>0$. Let us briefly review the main features of this result. The proof is an extension of the Mermin-Wagner theorem [7] and relies on the use of a Bogoliubov inequality [8] to bound the square of the magnetization from above.

Consider a statistical theory with a hamiltonian as that given in (2.2) defined on a $d$-dimensional hypercubic lattice. To analyze the symmetry structure of such a theory we follow the general procedure used to prove the Mermin-Wagner theorem by making use of a Bogoliubov inequality,

$$
\frac{1}{2} \beta\left\langle\left\{A, A^{\dagger}\right\}\right\rangle\left\langle\left[[C, H], C^{\dagger}\right]\right\rangle \geqslant|\langle[C, A]\rangle|^{2},
$$

where a dagger stands for hermitian conjugation, and $[],,\{$,$\} denote, respectively,$ a commutator and an anticommutator. $\langle O\rangle$ represents the thermal average of the operator $O$ at inverse temperature $\beta$. Calling the state of the system defined by the set $\{\phi(x)\}$ for all points, $x$, on the lattice, $|\phi(x)\rangle$, we can define suitable operators $A$ and $C$ through their action on these states in such a way that (2.3) leads to an inequality bounding the magnetization. One such set of operators is given by

$$
\begin{aligned}
& C_{k}|\phi(x)\rangle=|\phi(x)-\delta \phi \cos k \cdot x\rangle+|\phi(x)-\delta \phi \sin k \cdot x\rangle, \\
& A_{k}|\phi(x)\rangle=\sum_{y} \cos k \cdot y \sin \phi(y)\left|\Phi_{1}(x)\right\rangle+\sum_{y} \sin k \cdot y \sin \phi(y)\left|\Phi_{2}(x)\right\rangle,
\end{aligned}
$$


where

$$
\begin{aligned}
& \left|\Phi_{1}(x)\right\rangle \equiv|\phi(x)+\delta \phi \cos k \cdot x\rangle, \\
& \left|\Phi_{2}(x)\right\rangle \equiv|\phi(x)+\delta \phi \sin k \cdot x\rangle .
\end{aligned}
$$

In the above expressions $\delta \phi$ is a small constant field and $\boldsymbol{k}$ is a momentum vector in the first Brillouin zone. Consider the case $n=2$. To study the possibility of a spontaneous breaking of the global symmetry, we consider a hamiltonian $H_{0}(\phi)$, invariant under (2.1) with $n=2$ and add a symmetry-breaking term in the form of an interaction with a constant, external magnetic field $h$ :

$$
H=H_{0}(\phi)-h \sum_{x} \cos \phi(x) .
$$

Now, if $H_{0}(\phi)$ is invariant under (2.1), it is easy to prove that it can be written in the form (2.2) with each of the $f_{p}$ separately invariant under the same transformation, so that

$$
H_{0}(\phi)=\sum_{x} \sum_{p=1}^{s} f_{p}\left(\sum_{j=1}^{q(p)} c_{p j} \phi_{j}\right) .
$$

Assuming that the $f_{p}$ are expandable in a Taylor series about the zero of their arguments, use of the above operators in (2.7) leads to an upper bound for the square of the magnetization, $m$,

$$
m^{2} \leqslant \beta N\left[\sum_{k} \frac{1}{\gamma \sum_{p} \varepsilon_{p}+h m}\right]^{-1},
$$

where $N$ is the number of sites in the lattice, $\gamma$ is a positive number which bounds the thermal average of $f_{p}^{\prime \prime}$ from above, and

$$
\varepsilon_{p}=\left[\sum_{i=1}^{q(p)} c_{p l} \cos k \cdot \boldsymbol{r}_{t}\right]^{2}+\left[\sum_{t=1}^{q(p)} c_{p l} \sin k \cdot \boldsymbol{r}_{1}\right]^{2} .
$$

Note that we are still dealing with a classical theory; bras and kets are row and column vectors in $\boldsymbol{x}$ space and operators are matrices which act on this space.

We will now show that if $H_{0}$ is invariant under the transformation

$$
\phi\left(x_{1}, \ldots, x_{d}\right) \rightarrow \phi\left(x_{1}, \ldots, x_{d}\right)+\Lambda\left(x_{3}, \ldots, x_{d}\right),
$$

then the sum over $k$ on the right-hand side of (2.9) diverges in the thermodynamic limit as $h \rightarrow 0$, thus implying that the magnetization vanishes in this limit.

Since each of the $f_{p}$ are separately invariant under (2.10), for each $p$ the coefficients appearing in the arguments of the $f_{p}$ (see (2.2)) satisfy

$$
\sum_{J \in \alpha} c_{p j}=0
$$


where $\alpha$ represents the set of all spin labels which lie in a given $\left(x_{1}, x_{2}\right)$ plane. (In continuum notation this is equivalent to the statement that $H_{0}$ depends on $\phi$ only through $\partial_{1} \phi$ and $\partial_{2} \phi$. This allows terms in $H_{0}$ such as $\left(\partial_{3} \partial_{2} \phi\right)^{2}$, but not terms such as, for example, $\left(\partial_{3} \phi\right)^{2}$.)

Because $\varepsilon_{p}=0$ for $k_{1}=k_{2}=0$ the fact that it has a double Taylor expansion in powers of $k_{1}$ and $k_{2}$ implies that, in general,

$$
\varepsilon_{p}=k_{1}^{2} u_{p}(\boldsymbol{k})+k_{2}^{2} v_{p}(\boldsymbol{k})+k_{1} k_{2} w_{p}(\boldsymbol{k}),
$$

where $u_{p}, v_{p}$ and $w_{p}$ are finite functions of $k$. Consider a large lattice of linear dimension $L$. Defining $u(k)=\gamma \sum_{p} u_{p}(k)$, etc., and converting the sum over $k$ in (2.9) into an integral leads to

$$
m^{2} \leqslant \frac{(2 \pi)^{d} \beta}{I_{d}(L, h ; m)}
$$

where

$$
I_{d}=\int_{1 / L} \mathrm{~d}^{d} k\left[k_{1}^{2} u+k_{2}^{2} v+k_{1} k_{2} w+h m\right]^{-1} .
$$

Since $\varepsilon_{p} \geqslant 0$ for each $p$, looking at the region of integration where $k_{1}$ and $k_{2}$ are close to $1 / L$, it is clear that $I_{d}$ diverges at least as fast as $\ln L$ in the limit as $h \rightarrow 0$. Similarly, this integral will also diverge if the large- $L$ limit is taken first. Thus, in the thermodynamic limit $m^{2} \rightarrow 0$ as $h \rightarrow 0$ and the system has no long-range order: The global symmetry cannot be spontaneously broken. Contrasting the above arguments with those used to prove the usual Mermin-Wagner theorem shows clearly that the essential factor leading to the absence of long-range order is not the dimensionality of the system, but rather the dimensionality of the support over which the symmetry is global. It is trivial to apply the above arguments to prove that there is also no long-range order in an $n=1$ theory. To do so, it is sufficient to notice that, when $n=1, \varepsilon_{p}$ can be written just as for $n=2$ but with $v_{p}=w_{p}=0$. In this case the divergence of $I_{d}$ in the thermodynamic limit is at least linear.

Not only is the global symmetry unbroken for $n \leq 2$, but in fact, the full semi-local symmetry is also unbroken in this class of theories. To demonstrate this, let us again concentrate on the $n=2$ case; it is simple to construct a similar argument for $n=1$.

To test the possibility of breaking the semi-local symmetry, we need to construct an operator which will only break this symmetry, leaving other subsymmetries of the full $n=2$ symmetry, particularly the global one, intact. With $H_{0}$ invariant under (2.11), one can easily see that the relevant object to test is the two-point function defined by

$$
\Gamma_{M}=\cos [\phi(x)-\phi(x+M)],
$$

where $M$ is some vector with a non-zero projection out of the $\left(x_{1}, x_{2}\right)$ plane. We 
thus consider the hamiltonian

$$
H=H_{0}-h_{M} \sum_{x} \Gamma_{M}(x)
$$

and compute

$$
m_{M}=\left\langle\sum_{x} \Gamma_{M}(x)\right\rangle .
$$

As we did for the case of global symmetry breaking, we will test the possibility of breaking the semi-local symmetry by computing (2.17) using (2.16) and then taking the limit as $h_{M}$ tends to zero. A non-vanishing value of $m_{M}$ in this limit would signal the spontaneous breaking of the semi-local symmetry. To construct an upper bound on $m_{M}^{2}$, we again use a Bogoliubov inequality with $C_{k}$ defined as in (2.6) but with

$$
\begin{aligned}
A_{k}|\phi(x)\rangle= & \sum_{y}(\cos k \cdot y-\cos k \cdot(y-M)) \Delta_{M}(\phi(y))\left|\Phi_{1}(x)\right\rangle \\
& +\sum_{y}(\sin k \cdot y-\sin k \cdot(y-M)) \Delta_{M}(\phi(y))\left|\Phi_{2}(x)\right\rangle,
\end{aligned}
$$

where

$$
\Delta_{M}(\phi(y)) \equiv \sin (\phi(y)-\phi(y+M)) .
$$

It is now straightforward to compute (2.17). We find [2]

$$
\begin{gathered}
m_{M}^{2} \leqslant \frac{(2 \pi)^{d} \beta}{4}\left[\int_{1 / L} \mathrm{~d}^{d} k \frac{\xi_{M}^{2}(k)}{k_{1}^{2} u(k)+k_{2}^{2} v(k)+k_{1} k_{2} w(k)+\xi_{M}(k) h_{M} m_{M}}\right]^{-1}, \\
\xi_{M}(k)=1-\cos k \cdot M .
\end{gathered}
$$

Since $M$ has a component out of the $\left(x_{1}, x_{2}\right)$ plane, the numerator of the integrand in (2.19) does not vanish when $k_{1}=k_{2}=0$. Because the integrand in (2.19) is positive, the analysis proceeds as for (2.15) and the divergences are similar. Thus, $m_{M}^{2}=0$ in the thermodynamic limit and the full $n=2$ symmetry is not spontaneously broken.

Notice that it is essentially the same mechanism which is responsible for the absence of spontaneous magnetization in both of the cases we have analyzed: it is the same long-wavelength phonon excitations which prevent both the local as well as the semi-local symmetries from being broken. One can apply similar arguments to test for symmetry-breaking in other directions, and symmetry-breaking associated with higher multibody correlation functions in this and related theories. An example of a theory in which the semi-local symmetry is related to a four-body correlation has been discussed in the literature [5] and will be briefly analyzed in the next subsection.

Before proceeding to the next section, we make one important extension of our theorem. For a theory with $n=1$, the global symmetry cannot be spontaneously broken, whether the internal symmetry is continuous or discrete. As we shall show in sect. 3 , this is because an $n=1$ theory in $d$ dimensions can be transformed into a set of non-interacting systems in $d-1$ dimensions in a manner similar to the way 
in which the one-dimensional Ising model can be rewritten as a set of non-interacting spins. Here again we see how a system with a symmetry index $n$ and $d$ dimensions behaves qualitatively like an ordinary $n$-dimensional theory with global symmetry.

\subsection{GENERALIZATIONS OF INDEX-n SYMMETRIES}

The symmetries defined in (2.1) can be generalized in a natural and important way. The space-dependence of $\Lambda$ in (2.1) can be expressed by saying that $\Lambda$ is a function of all $d$ coordinates, but is constrained to satisfy

$$
\Delta_{1} \Lambda=\Delta_{2} \Lambda=\cdots=\Delta_{n} \Lambda=0,
$$

where $\Delta_{i}$ is a finite-difference operator in the $x_{i}$ direction. Such a set of conditions can be generalized to

$$
O_{1} \Lambda=O_{2} \Lambda=\cdots=O_{n} \Lambda=0,
$$

where the $O_{1}$ are a set of $n$ linearly independent difference (or, in continuum language, differential) operators. Theories constructed consistent with the conditions given in (2.21) will generally have interactions defined on lattice elements other than simple hypercubes.

The class of theories with such a suitably defined extended index- $n$ symmetry is expected to share many of the properties of theories with an ordinary index $-n$ symmetry. Among the most important of these is the absence of long-range order for $n=1$ or 2. It is not difficult to see how this result generalizes to include these cases as well. Recall that we argued that, in the restricted case of transformations of the type given in (2.11), all the terms in $H_{0}$ depended on $\phi$ only through $\partial_{1} \phi$ and $\partial_{2} \phi$ which implied that the inverse propagator in momentum space was necessarily of the form given in (2.13). In much the same way, there are a large class of operators, $O_{1}$ and $O_{2}$ such that symmetry of $H_{0}$ under (2.21) implies that it can only depend on $\phi$ through $O_{1} \phi$ and $O_{2} \phi$. This in turn implies a structure for $\varepsilon_{p}$ similar to (2.13) but in which the role of $k_{1}^{2}$ and $k_{2}^{2}$ will be taken by some other quadratic combination of the momenta, reflecting the form of the operators $O_{1}$ and $\mathrm{O}_{2}$. There will then be many cases in which a bound on the square of the magnetization (or some other $n$-point function), similar to those we obtained above, will indicate the absence of symmetry-breaking. It would be worthwhile to discover the precise, general conditions on the $O_{t}$ required to lead to this conclusion. An example of an $n=2$ theory invariant under a transformation of the form (2.21) which has no symmetry breaking [4] will be briefly discussed below.

\subsection{MISCELLANEOUS COMMENTS}

(i) It is clearly reasonable to believe that a result similar to the theorem of subsect. 2.2 can be proven in the case of non-abelian symmetries, since one generally expects that disorder will come about more easily when the number of degrees of freedom is increased. It is also clear that there may be exceptions to this simple intuitive 
argument, but, for an appropriately defined class of non-abelian theories with $n=1$ or 2 it should be possible to prove an analogous theorem.

(ii) It should be possible to construct theories, by a variety of methods, with more than one field, and with semi-local symmetries of the type we have discussed here. In this way, it should be possible to discuss the absence of symmetry-breaking in a wide variety of theories ranging from simple $d=2$ globally-symmetric theories to bona-fide locally-symmetric gauge theories in any dimension. With this approach one may be able to discuss both the Mermin-Wagner theorem and Elitzur's theorem [9] in the same framework and probe for a deeper connection between them which is presently not apparent.

(iii) Throughout the above discussion we have been somewhat cavalier about problems which might arise as a result of boundary conditions. It is clear that this is an important point which has to be addressed. The relevance of boundary conditions becomes apparent when one observes that a term in the hamiltonian of the form $\sum_{x} \partial_{1} \phi(x) \partial_{2} \phi(x)$ is invariant under (2.11) while a term such as $\sum_{x} \phi(x) \partial_{1} \partial_{2} \phi(x)$ is not. Clearly, a more precise statement of our theorem and the conditions under which it is valid must account properly for boundary effects. Boundary conditions are also clearly relevant in the context of the representation of these theories in terms of their topological excitations, as we will see when we discuss specific models in the following section. In the case of theories with an $n=1$ or 2 symmetry, it frequently happens that the topological excitations interact through very long range potentials; logarithmic or even worse. In cases such as these, boundary conditions may introduce global, topological as well as energetic constraints on the allowed configurations.

\section{Specific models}

\subsection{THREE SIMPLE MODELS WITH $n \leqslant 2$}

Most of the relevant properties of theories with uncommon symmetries of the type we have been talking about can be encountered in very simple models. In this section we shall mention three such theories and enumerate their salient features. For clarity, the simplest of these models will be discussed in some detail.

Before plunging into a discussion of the models themselves, it is convenient to define an interaction term, $I_{r}$, associated with the $2^{r}$ sites of an $r$-dimensional hypercube in a $d$-dimensional hypercubic lattice with $r \leqslant d$. With the spins, $s(r)$, defined on the lattice, we have

$$
\begin{aligned}
I_{r}\left(i ; x_{1}, x_{2}, \ldots, x_{r}\right) \equiv & \frac{1}{2} s(i) s^{\dagger}\left(i+\hat{x}_{1}\right) s^{\dagger}\left(i+\hat{\boldsymbol{x}}_{2}\right) \\
& \cdots s^{\dagger}\left(i+\hat{\boldsymbol{x}}_{r}\right) s\left(i+\hat{\boldsymbol{x}}_{1}+\hat{\boldsymbol{x}}_{2}\right) s\left(i+\hat{\boldsymbol{x}}_{1}+\hat{\boldsymbol{x}}_{3}\right) \\
& \cdots s^{(\dagger)}\left(i+\sum_{J=1}^{r} \hat{\boldsymbol{x}}_{j}\right)+\text { h.c. }
\end{aligned}
$$


where $i$ labels a lattice site and $\hat{\boldsymbol{x}}_{\boldsymbol{j}}$ is the unit vector in the $j$ direction. The last factor in (3.1) is complex-conjugated if $r$ is odd and is not when $r$ is even.

The first and simplest model we shall discuss is an index-2, three-dimensional theory, the so-called $2 / 24$ model $^{\star}$. Its hamiltonian is given by

$$
H=\alpha_{1} \sum_{t} I_{1}(i ; z)+\alpha_{2} \sum_{1} I_{2}(\imath ; x, y),
$$

where $I_{1}$ and $I_{2}$ are defined by (3.1) and the spins, $s(r)$, are U(1) (or $Z_{n}$ ) variables of the form

$$
S(\boldsymbol{r})=\mathrm{e}^{i \phi(r)} .
$$

In the $Z_{N}$ case, the phase of $s(r)$ takes the values

$$
\phi(\boldsymbol{r})=\frac{2 \pi}{N} p(\boldsymbol{r}), \quad p(\boldsymbol{r})=0,1, \ldots, N-1 .
$$

The interactions are thus a simple two-body term in the $z$-direction, and a four-body term along an elementary plaquette in the $x-y$ plane (see fig. 1). It is easy to see that this theory is invariant under

$$
\phi(x, y, z) \rightarrow \phi(x, y, z)+\lambda_{1}(x)+\lambda_{2}(y),
$$

where $\lambda_{1}$ and $\lambda_{2}$ are arbitrary functions of their arguments. There are, therefore, two distinct $n=2$ symmetries: $H$ is invariant under a uniform rotation of all spins which lie in any given $y-z$ plane or in any given $x-z$ plane. because $\lambda_{1}(x)+\lambda_{2}(y)$ is not an arbitrary function of $x$ and $y$, the theory does not have an $n=1$ symmetry.

Let us rewrite (3.2) explicitly for the case of $Z_{N}$ spins:

$$
H=\alpha\left[\sum_{L} \cos \Delta_{2} p(\boldsymbol{r})+\sum_{P} \cos \Delta_{4} p(\boldsymbol{r})\right],
$$

where

$$
\Delta_{2} p(\boldsymbol{r}) \equiv \frac{2 \pi}{N}[p(\boldsymbol{r}+\hat{z})-p(\boldsymbol{r})]
$$

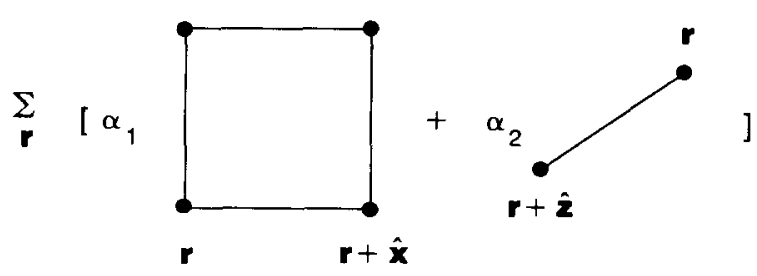

Fig. 1. The hamiltonian of eq. (3.3a)

* This model is sometimes referred to as the February 24 model 


$$
\Delta_{4} p(\boldsymbol{r}) \equiv \frac{2 \pi}{N}[p(\boldsymbol{r})-p(\boldsymbol{r}+\hat{\boldsymbol{x}})+p(\boldsymbol{r}-\hat{\boldsymbol{x}}+\hat{\boldsymbol{y}})-p(\boldsymbol{r}+\hat{\boldsymbol{y}})],
$$

where $\hat{\boldsymbol{x}}, \hat{\boldsymbol{y}}$ and $\hat{z}$ represent unit vectors, and $p=0,1, \ldots, N-1$. For simplicity, we have specialized our discussion to the case, $\alpha_{1}=\alpha_{2}=\alpha$. The first sum in (3.2) is over links in the $z$-direction $(L)$ and the second over all elementary plaquettes in each $x-y$ plane $(P)$ of the lattice. In what follows we assume the system is defined on a lattice with periodic boundary conditions.

The theory defined by (3.3) is self-dual in the sense that its periodic gaussian or villain-like formulation is self-dual. Further, interactions of the dual form of the full hamiltonian (3.3) are of the same form as those appearing in (3.3). There are, in addition, higher harmonics of those interactions [10]. A generalization of this theory which included all such harmonics would be self-dual in the usual sense.

To discuss the duality properties of (3.3) more completely, it is useful to study a periodic gaussian version of the model. The partition function for the periodic gaussian version of (3.3) is given by

$$
\begin{aligned}
Z(\beta) & =\sum_{\{p(\boldsymbol{r})=-\infty\}}^{\infty} \sum_{\left\{K_{P}=-\infty\right.}^{\infty} \sum_{\left\{K_{L}=-\infty\right\}}^{\infty} \\
& \times \exp \left\{-\frac{1}{2} \beta\left[\sum_{L}\left[\Delta_{2} p(\boldsymbol{r})-2 \pi K_{L}\right]^{2}+\sum_{P}\left[\Delta_{4} p(\boldsymbol{r})-2 \pi K_{P}\right]^{2}\right]\right\},
\end{aligned}
$$

where we have introduced the integer fields $K_{L}$ and $K_{P}$ attached to links in the $z$-direction and to plaquettes in $x-y$ planes, respectively. For convenience, we have extended the sum over $p(\boldsymbol{r})$ to go from $-\infty$ to $\infty$. This introduces a uniform and harmless overcounting [11] which will not concern us here.

To construct the dual of (3.4) we use the Poisson identities

$$
\begin{aligned}
\sum_{p(r)=-\infty}^{\infty} F[p(\boldsymbol{r})] & =\sum_{l=-\infty}^{\infty} \int_{-\infty}^{\infty} \mathrm{d} \eta \delta(\eta-l) F(\eta) \\
& =\sum_{m=-\infty}^{\infty} \int_{-\infty}^{\infty} \mathrm{d} \eta F[\eta] \mathrm{e}^{2 \pi l m \eta}
\end{aligned}
$$

to obtain

$$
\begin{aligned}
Z(\beta)= & \sum_{\{\kappa(\boldsymbol{r})=-\infty\}\left\{K_{L}, K_{P}=-\infty\right\}}^{\infty} \int_{-\infty}^{\infty} \Pi \mathrm{d} \eta(\boldsymbol{r}) \exp \left\{-\frac{1}{2} \beta\left[\sum_{L}\left[\Delta_{2} \eta(\boldsymbol{r})-2 \pi K_{L}\right]^{2}\right.\right. \\
& \left.\left.+\sum_{P}\left[\Delta_{4} \eta(\boldsymbol{r})-2 \pi K_{P}\right]^{2}\right]\right\} \exp \left(2 \pi i \sum_{S} \kappa(\boldsymbol{r}) \eta(\boldsymbol{r})\right),
\end{aligned}
$$

where the last sum (over $S$ ) is a sum over all sites of the lattice. In the above equation we have introduced an integer-valued field, $\kappa$, located at the lattice sites. We now introduce continuous $z$-link and $x-y$ plaquette fields, $\tau_{L}$ and $\tau_{P}$ In what 
follows these will be associated with the sites of the dual lattice. Dropping irrelevant overall multiplicative ( $\beta$-dependent but field-independent) factors, we obtain

$$
\begin{aligned}
Z(\beta)= & \sum_{\left\{K_{L}, K_{\beta} \kappa(r)=-\infty\right\}}^{\infty} \int \mathrm{D} \eta(\boldsymbol{r}) \int \mathrm{D} \tau_{L}(\boldsymbol{r}) \int \mathrm{D} \tau_{P}(\boldsymbol{r}) \\
& \times \exp \left[\sum_{L}\left[-\frac{1}{2 \beta} \tau_{L}^{2}+i \tau_{L}\left(\Delta_{2} \eta-2 \pi K_{L}\right)\right]\right. \\
& \left.+\sum_{P}\left[-\frac{1}{2 \dot{\beta}} \tau_{P}^{2}+i \tau_{P}\left(\Delta_{4} \eta-2 \pi K_{P}\right)\right]+\sum_{L} 2 \pi i \kappa(\boldsymbol{r}) \eta(\boldsymbol{r})\right] .
\end{aligned}
$$

To do the integral over $\eta$, we first integrate (sum) by parts the terms

$$
\sum_{L} \tau_{L} \Delta_{2} \eta=-\sum_{L} \eta(r) \Delta_{2} \tau_{L}\left(r-\frac{1}{2}\right)
$$

and

$$
\sum_{P} \tau_{P} \Delta_{4} \eta=\sum_{P} \eta(\boldsymbol{r}) \Delta_{4} \tau_{P}\left(\boldsymbol{r}-\frac{1}{2}(\hat{x}+\hat{y})\right) .
$$

The integral over $\eta$ can now be done, leading to a product of $\delta$-function constraints of the form

$$
\prod_{L} \delta\left[2 \pi \kappa(\boldsymbol{r})-\Delta_{2} \tau_{L}\left(\boldsymbol{r}-\frac{1}{2} \hat{\boldsymbol{z}}\right)+\Delta_{4} \tau_{P}\left(\boldsymbol{r}-\frac{1}{2}(\hat{\boldsymbol{x}}+\hat{\boldsymbol{y}})\right)\right] .
$$

To solve these constraints, we define a dual field, $\psi$, at the sites of the dual lattice, $\tilde{\boldsymbol{r}}=\boldsymbol{r}+\frac{1}{2}(\hat{\boldsymbol{x}}+\hat{\boldsymbol{y}}+\hat{z})$, in such a way that

$$
\begin{aligned}
\tau_{L}\left(\boldsymbol{r}+\frac{1}{2} \hat{z}\right) & =\frac{N}{2 \pi} \Delta_{4} \psi(\tilde{\boldsymbol{r}}-\hat{\boldsymbol{x}}-\hat{\boldsymbol{y}})+\tilde{J}_{P} N, \\
\tau_{P}\left(\boldsymbol{r}+\frac{1}{2}(\hat{\boldsymbol{x}}+\hat{\boldsymbol{y}})\right) & =\frac{N}{2 \pi} \Delta_{2} \psi(\tilde{\boldsymbol{r}}-\hat{z})+\tilde{J}_{L} N,
\end{aligned}
$$

where $\tilde{J}_{L}$ and $\tilde{J}_{P}$ are integer fields located on the $z$-links and $x-y$ plaquettes of the dual (shifted) lattice. We can replace the sums over $\tau_{L}$ and $\tau_{P}$ by sums over $\psi, \tilde{J}_{L}$ and $\tilde{J}_{P}$ [11]. The result, neglecting overall constants, is

$$
\begin{aligned}
Z= & \sum_{\left\{K_{L}, K_{P}\right\}} \sum_{\left\{\tilde{J}_{z}, \tilde{J}_{P}=-\infty\right\}}^{\infty} \int \mathrm{D} \psi \\
& \times \exp \left[\sum_{L}\left[-\frac{N^{2}}{8 \pi^{2} \beta}\left(\Delta_{4} \psi+2 \pi \tilde{J}_{P}\right)^{2}-i N K_{\mathrm{L}} \Delta_{4} \psi(\boldsymbol{r}-\hat{\boldsymbol{x}}-\hat{y})\right]\right. \\
& \left.+\sum_{P}\left[-\frac{N^{2}}{8 \pi^{2} \beta}\left(\Delta_{2} \psi+2 \pi \tilde{J}_{L}\right)^{2}-i N K_{P} \Delta_{2} \psi(\boldsymbol{r}-\hat{z})\right]\right],
\end{aligned}
$$

where the sums over $z$-links $(L)$ and $x-y$ plaquettes $(P)$ refer to the original lattice. 
However, it is easy to see that

$$
\begin{gathered}
\sum_{L} K_{L} \Delta_{4} \psi(\tilde{\boldsymbol{r}}-\hat{\boldsymbol{x}}-\hat{y})+\sum_{P} K_{P} \Delta_{2} \psi(\tilde{\boldsymbol{r}}-\hat{z})=\sum_{\tilde{\boldsymbol{r}}} \psi(\tilde{\boldsymbol{r}}) \frac{2 \pi}{N} m(\tilde{\boldsymbol{r}}), \\
m(\tilde{\boldsymbol{r}}) \equiv-\Delta_{2} K_{P}+\Delta_{4} K_{L} .
\end{gathered}
$$

This allows us to refer all sums in (3.7) to the dual lattice, with the result

$$
\begin{aligned}
Z= & \sum_{m(\tilde{\boldsymbol{r}})=-\infty}^{\infty} \sum_{\tilde{J}_{L}, \tilde{J}_{P}=-\infty}^{\infty} \int \mathrm{D} \psi \exp \left\{-\tilde{\beta}\left[\sum_{\tilde{\tilde{S}}}\left(\Delta_{4} \psi-2 \pi \tilde{J}_{P}\right)^{2}\right.\right. \\
& \left.\left.+\sum_{\tilde{\boldsymbol{P}}}\left(\Delta_{2} \psi-2 \pi \tilde{J}_{L}\right)^{2}+\sum_{\tilde{S}} 2 \pi i m(\tilde{\boldsymbol{r}}) \psi(\tilde{\boldsymbol{r}})\right]\right\}
\end{aligned}
$$

where we have defined a new coupling, $\tilde{\beta} \equiv N^{2} / 8 \pi^{2} \beta$.

Using (3.9), one can prove the self-duality of this model by performing the summations over the $\boldsymbol{m}(\tilde{\boldsymbol{r}})$. Alternatively, integrating over $\psi$ leads to a representation for the partition function in terms of point-like degrees of freedom embodied in the integer-valued field $m$. Let us first discuss the self-dual nature of the model. We shall then discuss its topological excitations.

Using the Poisson summation formula in (3.9) it is straightforward to prove that this version of the theory is self-dual. The result of this calculation is just (3.4) with the difference that $\beta$ has been replaced by $\tilde{\beta}$ and that the degrees of freedom are now defined on the dual lattice. As we have mentioned before, we have chosen to deal with the periodic gaussian approximation to our theory for simplicity. Indeed, following essentially the same steps used to derive (3.9) from (3.4), one can manipulate (3.3) to prove that that model is precisely self-dual. (That is, no extra harmonics of the interactions in $H$ are generated by the duality transformation for $N=2,3$ and 4.) This is entirely analogous to the structure found in the 2-dimensional $Z_{N}$ clock models. As is the case for those models, because of self-duality, if the theory has a single phase transition, the critical temperature must satisfy $\beta=\tilde{\beta}$. That this is true is demonstrated by our numerical analysis of the model for $N \leqslant 4$ discussed in the next subsection. For $N>4$ the model is not precisely self-dual, in that the dual theory includes harmonics of the original interactions as well, but its gaussian approximation, (3.4), is self-dual for all values of $N$. In this case, the self-dual point is given by $\beta=\tilde{\beta}=N / 2 \pi$. As our numerical analysis indicates, for $N \geqslant 5$, the model (3.3) has two phase transitions ${ }^{\star}$, one of which becomes essentially independent of $N$ as $N$ grows, and the other occurring at a temperature which approaches zero

* In fact, one can ngorously show that this must happen: As is true for the $d=2 \mathrm{Z}_{N}$ clock models, it is possible to derive a set of Griffiths-like inequalities for certain $n$-point correlation functions in our model (see ref. [5]). Using these inequalities, one can deduce the existence of three phases for the periodic gaussian approximation of the model It also follows from these rigorous inequalities that the intermediate phase which appears between ordered and disordered ferromagnetic phases has no long-range order, as expected from the general result which we proved in the last section 
like $N^{-2}$. This is consistent with the behavior expected for the self-dual gaussian version of the model (3.4), and is qualitatively similar to the behavior found for the $d=2$ clock models. It is important to stress the fact that self-duality of (3.4) is not determined by the particular operators which define the interactions of our model, nor does it have much to do with the spatial dimension or symmetry index of the model; indeed, there are three-dimensional $n=2$ models which are not self-dual (an example will be briefly described below). The self-duality of our model follows from the fact that the theory has two distinct types of interactions for a single field: Any theory satisfying these conditions will be self-dual in the sense described above. (A general discussion of this point can be found in ref. [12].)

Let us now discuss the topological excitations of the model. There are two types of integer-valued objects in this theory which may loosely be thought of as topological in origin. One is associated with the discreteness of the $Z_{N}$ symmetry and the other with its compactness. We are interested in studying the latter type, and to this end we will take the $U(1)$ limit of (3.9). We can write

where

$$
Z=\sum_{\{m(r)\}} \int \mathrm{D} \psi \exp (-H[\psi]),
$$

$$
-H[\psi]=\sum_{r}\left[-\frac{1}{2 \beta}\left(\frac{N}{2 \pi} \Delta_{2} \psi\right)^{2}-\frac{1}{2 \beta}\left(\frac{N}{2 \pi} \Delta_{4} \psi\right)^{2}+2 \pi i m(r) \psi(r)\right] .
$$

Transforming $H$ to momentum space and integrating over $\psi$ leads to a representation of the model in terms of point-like excitations represented by the field $m$ :

$$
Z=Z_{0} \sum_{\{m\}} \exp \left[-\beta \int \frac{\mathrm{d}^{3} k}{(2 \pi)^{3}}\left|m_{k}\right|^{2} D^{-1}(\boldsymbol{k})\right],
$$

where $Z_{0}$ summarizes the field-independent factors we have not been writing explicitly and the inverse propagator, $D(k)$, is given by

$$
D(k)=6-4 \cos k_{x}-4 \cos k_{y}+4 \cos k_{x} \cos k_{y}-2 \cos k_{z} .
$$

$D(k)$ has zeros inside the Brillouin zone, and so only those configurations in (3.12) for which $D^{-1}$ is finite will contribute to $Z$. The zeros of $D$ occur along the lines

$$
\begin{array}{ll}
k_{x}=k_{z}=0 & \text { for all } k_{y} \in \mathrm{B}, \\
k_{v}=k_{z}=0 & \text { for all } k_{x} \in \mathrm{B},
\end{array}
$$

where B is the first Brillouin zone, $[-\pi, \pi]$. Excluding configurations for which $D$ vanishes translates into a set of neutrality conditions for the charges $m(r)$. These can easily be seen to be given by

$$
\begin{array}{ll}
\sum_{\boldsymbol{r}} \mathrm{e}^{i y k_{y}} m(\boldsymbol{r})=0 & \text { for any } k_{x}, \\
\sum_{\boldsymbol{r}} \mathrm{e}^{i x k_{\mathrm{r}}} m(\boldsymbol{r})=0 & \text { for any } k_{y} .
\end{array}
$$


These conditions identify the configurations which contribute to the partition function as those for which the sum of topological charges, $\sum_{r} m(r)$, vanishes on each of the principal $x-z$ and $y-z$ planes of the lattice. This is a direct consequence of the compound $n=2$ symmetry of our model. Of course, since this symmetry contains a global U(1) symmetry, the above conditions imply, as they should, global neutrality of the $m$ field as well.

The complete partition function can therefore be written as

$$
Z=Z_{0} \sum_{\{m(r)\}} \exp \left(-\sum_{r, r^{\prime}} m(\boldsymbol{r}) G\left(\boldsymbol{r}, \boldsymbol{r}^{\prime}\right) m\left(\boldsymbol{r}^{\prime}\right)\right)
$$

where the sum over $\{m(r)\}$ includes only those configurations for which (3.15) is valid, and $G\left(r, r^{\prime}\right)$ is the configuration space propagator. The explicit form of the propagator can be obtained approximately by considering the dominant contributions of the Fourier transform of $D^{-1}$ close to the singular lines given by (3.14). The result is

$$
G\left(r, r^{\prime}\right) \sim \ln \left|x-x^{\prime}\right| \ln \left|y-y^{\prime}\right|-\frac{\left(z-z^{\prime}\right)^{2}}{2\left(x-x^{\prime}\right)^{2}\left(y-y^{\prime}\right)^{2}},
$$

where $s-s^{\prime}$ is the component of $\boldsymbol{r}-\boldsymbol{r}^{\prime}$ in the $s$ direction.

The similarity between (3.16) and the logarithmic gas representation of the two-dimensional $x-y$ model suggests that, just as the point charges in the $d=2 x-y$ model are the topological excitations of the model, so that $m(\boldsymbol{r})$ are topological excitations of the present theory. The interpretation of the point charges in the globally-symmetric $x-y$ model as representing the multiple windings of the U(1) spins around some closed contour is straightforward. The same is unfortunately not true in the present case. Recall, from (3.8), that the $m(\boldsymbol{r})$ are particular combinations of the integer fields $K_{P}$ and $K_{L}$ which we introduced to retain the periodicity of the hamiltonian in going from (3.3) to (3.4). This is still identical to the corresponding origin of the point charges in the $x-y$ model. However, here it is difficult to tell what are the configurations for the theory defined through (3.3) which lead to non-zero values for $m$. In the case of non-zero $K_{L}$, the interpretation is like that of the $x-y$ model: $K_{L}$ represents the number of complete rotations undergone by a spin as we move from a site at $r$ to the next in the $z$-direction. The interpretation of $K_{P}$ is not quite so simple, so that a geometrical interpretation of the $m$ 's is not entirely straightforward. It should be stressed that, as we have mentioned before [1], the fact that the energetics of the partition function are most simply expressed in terms of point charges (in this case), does not necessarily mean that the geometry of the topological excitations can be understood simply in this representation. It is quite possible that the $m(\boldsymbol{r})$ are related to some quality of a different object (e.g. the ends of a string), so that a complete understanding of the topological excitations may require considering topological objects of higher dimension. 


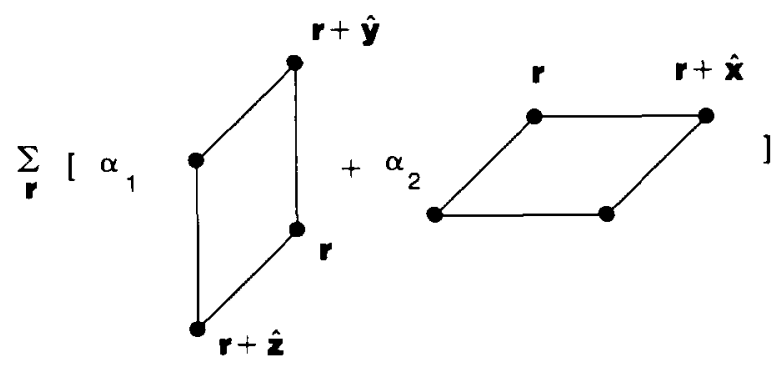

Fig. 2. The hamiltonian of eq. (318).

A second simple model which we would like to describe here is defined by the hamiltonian

$$
H=\sum_{i}\left[\alpha_{1} I_{2}(i ; x, z)+\alpha_{2} I_{2}(i ; y, z)\right],
$$

where the notation is the same as in (3.1); that is, the theory describes four-body interactions around elementary plaquettes in the $x-z$ and $y-z$ planes (see fig. 2). This theory is invariant under

$$
\phi(x, y, z) \rightarrow \phi(x, y, z)+\lambda_{1}(x, y)+\lambda_{2}(z),
$$

where the $\lambda_{t}$ are arbitrary functions of their arguments. The theory thus has a compound $n=2$ plus $n=1$ symmetry.

The appearance of an $n=1$ symmetry has radical consequences: It is not difficult to see that, because of this symmetry, the theory decouples into a set of noninteracting two-dimensional models. In fact, for the case of $Z_{N}(U(1))$ spins, (3.18) can be transformed into a set of decoupled $d=2 N$-state clock $(x-y)$ models. This is a general feature of theories with $n=1$ symmetries. Any such theory (when defined on the elementary simplices of the lattice) decouples along the direction defined by the $n=1$ axis. To see this, notice that the $n=1$ symmetry of this model allows us to perform global rotations of all spins which lie on any line along the $z$-direction. Therefore, by performing these linear transformations, any particular $x-y$ plane can be transformed so that all its spins have the same phase. Having fixed the gauge in this manner, the partition function for the model will factorize by the use of a Jordan-Wigner-like transformation which explicitly decouples the model.

The argument can be applied to any $n=1$ theory in $d$ dimensions to show that it will decouple into a set of non-interacting theories in $d-1$ dimensions. For the purpose of illustration, it is instructive to go through the argument for the onedimensional Ising model, a particularly simple $n=1$ theory.

Consider a one-dimensional Ising model in a finite lattice of $N$ spins with free boundary conditions. The partition function for this model is given by

$$
Z=\sum_{s_{1}} \sum_{s_{2}} \cdots \sum_{s_{N}} \mathrm{e}^{-\beta \sum_{1} s_{t} s_{t+1}},
$$


where $s_{t}= \pm 1$ is an Ising spin at site $i$. Define new Ising spins $\sigma_{\imath}$ such that

$$
s_{M}=\prod_{i=1}^{M} \sigma_{\imath} .
$$

It follows that $\sigma_{j}=s_{j} s_{j-1}$ for $j>1$, and $\sigma_{1}=s_{1}$. In terms of $\sigma_{t},(3.19)$ can be written as

$$
Z=\sum_{\sigma_{1} \sigma_{2}} \sum_{\sigma_{N}} \cdots \sum_{\sigma_{N}} \mathrm{e}^{-\beta \sum_{i=2}^{N} \sigma_{t}}=2(2 \cosh \beta)^{N-1} .
$$

It is straightforward to apply an analogous transformation to (3.18) along the $z$-direction to show that it is equivalent to a stack of two-dimensional $Z_{N}$ clock models. Furthermore, a generalization of the theorem of sect. 2 can be seen to follow from this non-local transformation: Because the $(d-1)$-dimensional theories into which the $d$-dimensional $n=1$ theory was transformed are non-interacting, the expectation value of the spin, $\langle s\rangle=0$. Therefore, as we mentioned in subsect. 2.2, theories with symmetry index, $n=1$ cannot break their global symmetry spontaneously, regardless of whether the group is discrete or continuous.

The third model we will present has a hamiltonian which can be written as

$$
H=\sum_{i}\left[\alpha_{1} I_{2}(i ; x, z)+\alpha_{2} I_{2}(i ; y, z)+\alpha_{3} I_{2}(i ; x, y)\right],
$$

where we have used the notation described in (3.1). The model thus describes four-body interactions of spins which lie at the corners of elementary plaquettes in the $x-z, y-z$ and $x-y$ planes (see fig. 3). This theory is invariant under the transformation

$$
\phi(x, y, z) \rightarrow \phi(x, y, z)+\lambda_{1}(x)+\lambda_{2}(y)+\lambda_{3}(z),
$$

and so, has a triple $n=2$ symmetry. Because of the last term in (3.22) though, it does not have the $n=1$ symmetry of (3.18).

Using the techniques of ref. [13], it is straightforward, if tedious, to perform the same analysis for this model as was done for the $2 / 24$ model. It is not difficult to see, however, in what ways these two models differ in their properties under a duality transformation. The fundamental interactions in (3.22) are defined on the

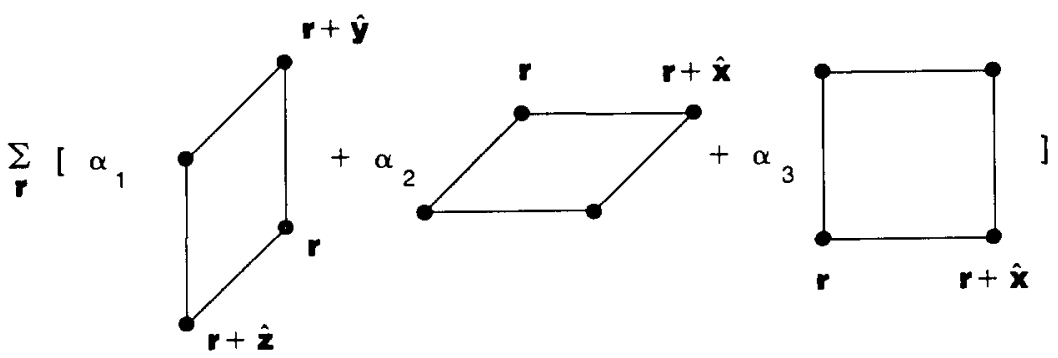

Fig. 3. The hamiltonian of eq. (3.22). 
plaquettes of the lattice. To each plaquette in the original theory there corresponds a link, $u_{\mu}$ in the dual theory, passing through the center of the original plaquette. The delta-function constraints which follow from the integration over angles require that the sum of the $12 u_{\mu}$ 's which form the edges of the cube surrounding the original lattice site vanish. These constraints are solved by defining integer link fields $v_{\mu}$ such that $u_{x}=\nabla_{x}\left(\nabla_{y} v_{y}-\nabla_{z} v_{z}\right)$ and so on for the other two directions. The $v_{\mu}$ 's therefore live on links forming crosses perpendicular to the corresponding $u_{\mu}$. Defining $\delta_{z} \equiv \nabla_{x} \nabla_{y}$, etc., the dual hamiltonian can be written in the form

$$
\begin{gathered}
H=\sum_{\mu v} F_{\mu v}^{2}, \\
F_{\mu v}=\delta_{\mu} v_{v}-\delta_{v} v_{\mu} .
\end{gathered}
$$

The symmetry under transformations of the $v_{\mu}$ is of the form

$$
v_{\mu} \rightarrow v_{\mu}+\delta_{\mu} \Lambda(\boldsymbol{r})
$$

Therefore, the dual theory is a local gauge theory. One important reason for presenting this example is that, although this three-plaquette model seems to be fundamentally different from the $2 / 24$ model, and certainly has a different duality structure, the phase structure of both theories is qualitatively the same. Therefore, there is no simple correspondence between the duality properties of these models and their phase structure.

\subsection{MONTE CARLO SIMULATIONS}

We have performed more or less extensive numerical analysis of all the above models. Details of the procedures which were used have been discussed in the literature [13]. Here we will briefly recapitulate the general strategy employed in our analysis and will state the results.

An overall view of the phase structure of the models was obtained by simulating thermal cycles. Starting from an ordered configuration at a large value of the inverse temperature, $\beta_{0}$, a thermal cycle is simulated by performing a succession of Monte Carlo iterations of the lattice and increasing the temperature before starting the next iteration. This procedure is repeated until $\beta=0$ and then reversed until $\beta=\beta_{0}$. Several quantities of interest are measured for each iteration. Although in such a numerical experiment the system is never really in equilibrium, it is generally close to equilibrium as long as the system does not go through a phase transition. In the neighborhood of a transition, however, the distance from equilibrium increases, and the measured quantities will show hysteresis-like loops. For the $Z_{N}$ version of these models, this type of analysis is done for several values of $N$. Examples of the internal energy of the $2 / 24$ model for $N=4,5,6,7,8$ and 9 are shown in figs. 4-9. Whereas the model for $N=4$ displays a single pronounced loop, as $N$ increases through 5 , the appearance of an intermediate region between two loops becomes evident. Once 


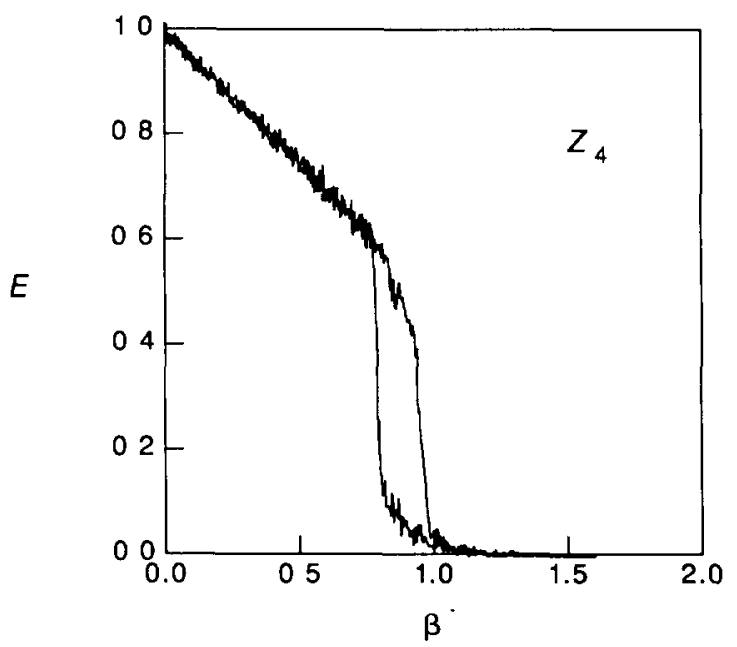

Fig. 4. The internal energy in a thermal cycle for the model defined in (3.4) for $N=4$. A single, pronounced loop is seen to occur at the model's self-dual point $\beta=\ln \left(1+2^{1 / 2}\right)=0.88$... Further analysis shows that this is a first-order transition. The lattıce size for this simulation measured $15 \times 15 \times 15$ with periodic boundary conditions. The step size in $\beta$ was $\delta \beta=0.001$. All simulations were done using a heat bath updating algorithm

these regions of interest have been identified through this procedure, a different type of simulation should be used to determine the type of transition (in the infinite-volume limit) which can be associated with a given hysteresis loop. The pattern which is seen to emerge in all cases is that these models undergo two transitions for $N \geqslant N_{\mathrm{c}}$. The high-temperature transition soon becomes independent

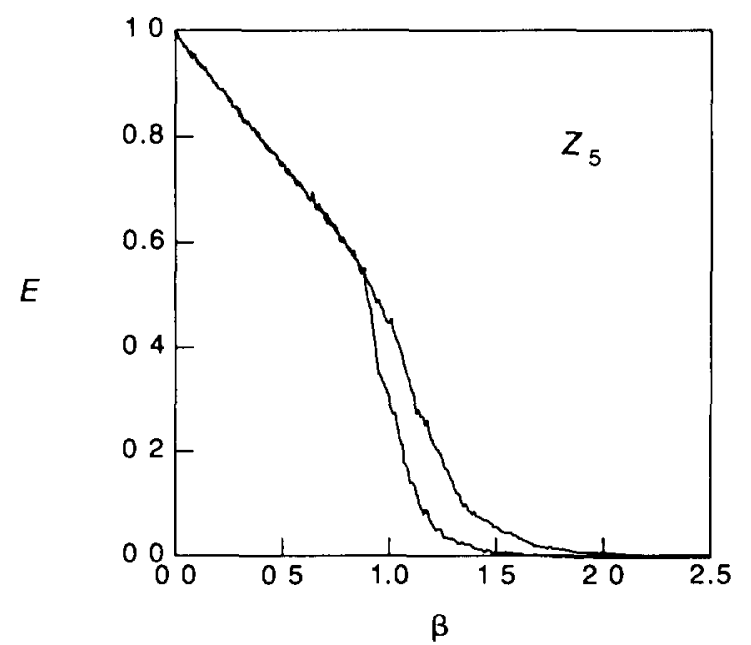

Fig. 5 Same as fig. 4 for $N=5$. A less pronounced loop than that seen for $N=4$. Further analysis points to two continuous nearby transitions at inverse temperatures $\beta_{1} \approx 0.9$ and $\beta_{2} \approx 1.1$. For this simulation. as well as for those pictured in figs. $6-9$, we used cubic lattices of linear dimension $L=20$ with periodic boundary conditions. The step size in $\beta$ was $\delta \beta=0002$. 


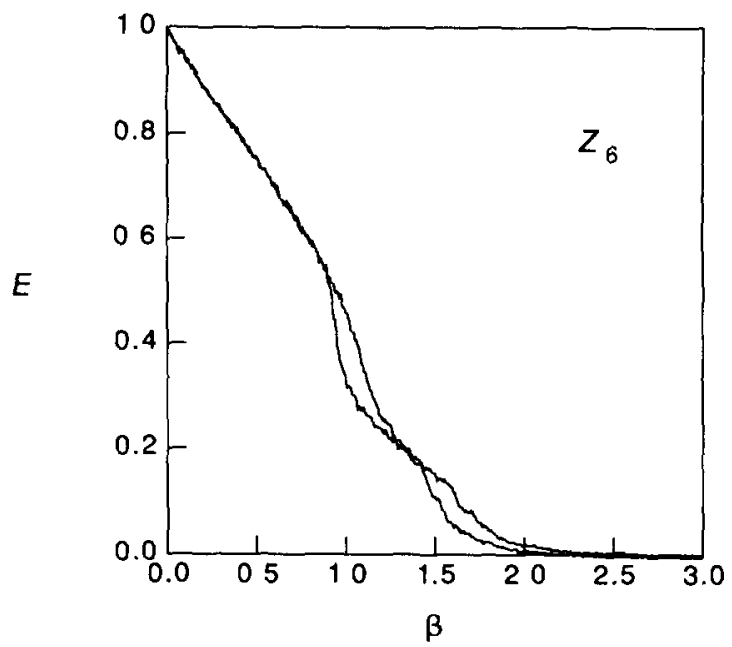

Fig. 6. Same as fig 5 for $N=6$. Two clearly separated loops and the appearance of an intermediate phase. Further analysis points to two contınuous transitions at inverse temperatures $\beta_{1} \approx 1.0$ and $\beta_{2} \approx 1.5$.

of $N$ as $N$ grows, in agreement with general arguments. The low temperature transition scales with $N$ as $\beta_{\mathrm{c}}=\gamma /[1-\cos (2 \pi / N)]$, with a value for the constant, $\gamma$, which depends on the details of the model. For the $2 / 24$ model, $\gamma \approx 0.75$.

When the transition is first order, the internal energy is discontinuous at the critical temperature. This permits a very precise determination of the critical temperature in these cases. By preparing an initial state in which half of the lattice is

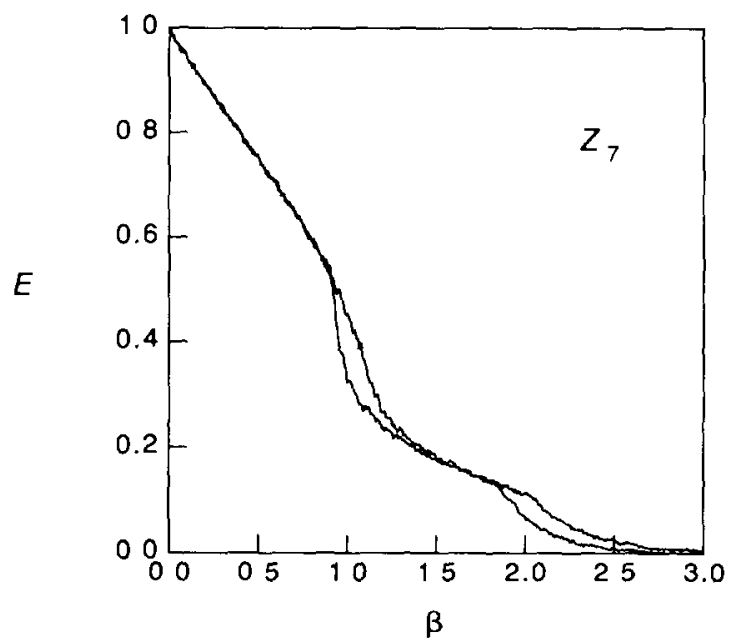

Fig. 7. Same as fig. 5 for $N=7$. Analyses performed in the critical regions points to two continuous transitions at inverse temperatures $\beta_{1}=1.0$ and $\beta_{2}=2.0$. In accordance with general principles and our discussion of sect 3 , the second transition is seen to move toward $\beta \rightarrow \infty$, as $N$ grows The lengthening intermediate phase can be seen to be dominated by massless, spin-wave excitations. 


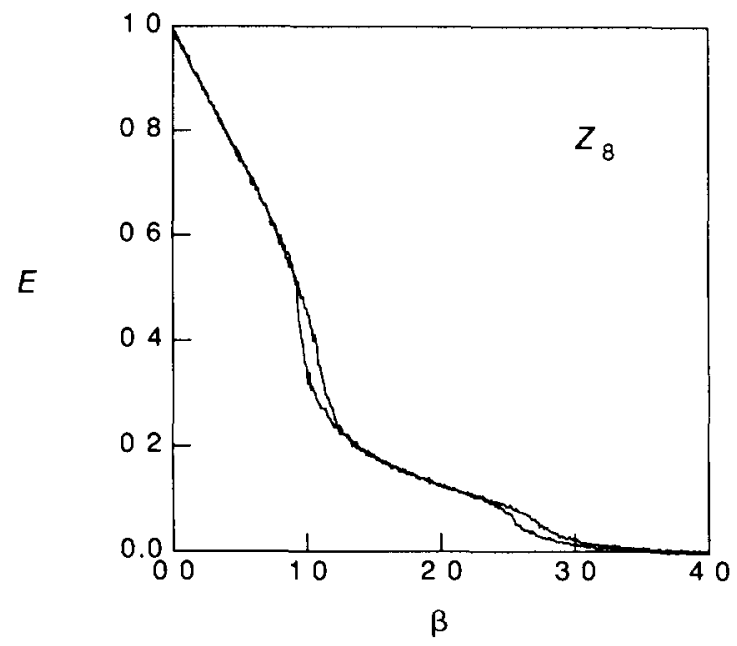

Fig. 8. Same as fig. 5 for $N=8$.

ordered and half of it is completely disordered, simulations are performed at a set of temperatures in the region of hysteresis and the time-evolution of the initial state is observed. At the critical temperature in a first-order transition, each half of the lattice will evolve into a different stable state, and the value of, say, the energy will appear to be roughly constant in time. At temperatures away from the critical point, however, the system rapidly evolves into a unique stable state. Once a good estimate of the critial temperature has been obtained in this manner, it is possible to distinguish fairly easily between first-order and continuous transitions by observing the time evolution of two extreme initial states, one completely disordered and one ordered,

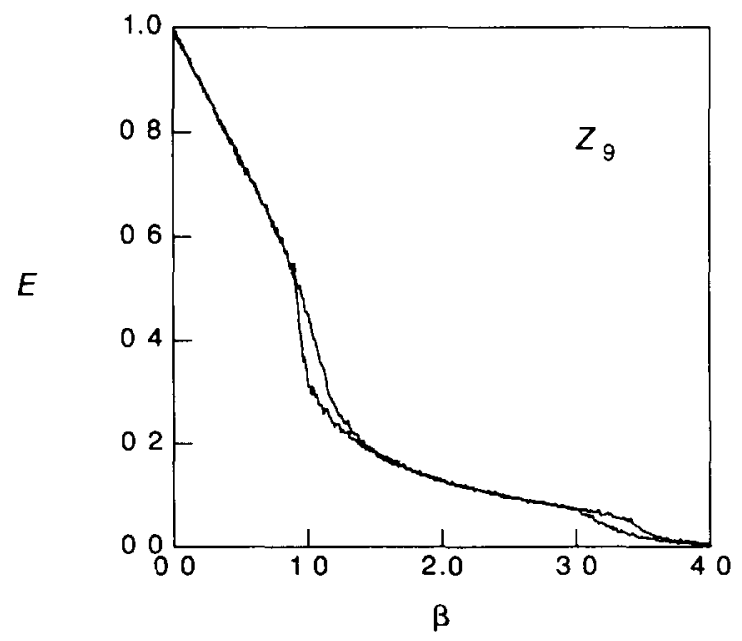

Fig. 9 Same as fig. 5 for $N=9$. 
at the estimated critical point. In a continuous transition, after a period of relaxation, the values of the energy for both states will coincide. At a first-order transition, on the other hand, the energy of the two states will evolve to two different values. Using this kind of simulation, we determined that for all models, with the exception of that given by (3.18), the transitions observed for $N \leqslant 4$ are first order. All other transitions observed are continuous. No effort was made to determine the order of the continuous transitions.

Further analysis to determine the nature of the intermediate phase observed for $N \geqslant N_{\mathrm{c}}$ along the lines discussed in ref. [5] was performed for these models. The results are consistent with the absence of long-range order in the intermediate phase and therefore with the absence of long-range order in the $U(1)$ limit, in accordance with the prediction of the theorem of sect. 2, and the behavior of two-dimensional $\mathrm{Z}_{N}$ clock models.

It is interesting to note that the value of $N_{\mathrm{c}}$ is the same for all three models studied in this section and coincides with the value found in two-dimensional clock models. All these models share the same type of index- $n$ symmetry; that is, the constraints on $\Lambda$ are all of the type shown in (2.20). In the next section we will describe an $n=2$ theory whose gauge function satisfies constraints of a different form. We will find that the qualitative phase structure of that theory is similar to that of those satisfying (2.20), but with a different value of $N_{\mathrm{c}}$.

\section{MORE GENERAL MODELS}

The models we have discussed so far share two important characteristics: They are all defined on cubic lattices and they all have index- $n$ symmetries in the restricted sense of (2.20). In this section we will briefly discuss two theories which differ in these characteristics. We will be particularly interested in finding out whether the phase structure of these theories is qualitatively similar to the ones we have studied above and, if so, whether the critical value of $N$ is the same.

The first model is a simple but interesting statistical theory of $Z_{N}$ spins defined on a three-dimensional FCC lattice [6]. The model is described by the hamiltonian

$$
H=\alpha \sum_{\tau} \cos \Delta_{\tau}(\phi) .
$$

where $\Delta_{\tau}(\phi)=(2 \pi / N)\left(\phi_{1}+\phi_{2}-\phi_{3}-\phi_{4}\right)$. The sum in (3.24) is over all elementary tetrahedra of the lattice, and the indices on $\phi$ refer to the four vertices of a given tetrahedron. $H$ has compound $n=2$ symmetries of the type (2.20) and is self-dual in the sense we have employed above. As $N \rightarrow \infty$, the model has point-like topological excitations with interactions which are basically logarithmic. For finite $N$ there are also domain-wall-like excitations. The phase structure of this model is quite similar to that of the two-dimensional clock models [3], the locally-symmetric $Z_{N}$ gauge theories [14], and the models described in subsects 3.1 and 3.2 above. For $N$ smaller 
than a critical value, $N_{c}$, the model has a single, first-order transition separating an ordered from a disordered phase (the corresponding transitions in the $Z_{N}$ gauge theory is also first order, whereas that of the $d=2$ clock models is second order). For $N \geqslant N_{\mathrm{c}}$ this transition bifurcates into two softer ones demarcating an intermediate phase with no long-range order. In the limit as $N \rightarrow \infty$, where the model has a $\mathrm{U}(1)$ symmetry, the low-temperature transition moves toward $T=0$ as $N^{-2}$, and the symmetry-breaking phase disappears, consistent with the theorem of the past subsection. For this model, as for the clock model in $d=2$ and the $Z_{N}$ gauge theory in $d=4$, as well as the models described in subsect. $3.1, N_{\mathrm{c}}$ appears to be close to 5. Another example in this category which has been discussed in the literature is described by a hamiltonian similar to (3.24) but defined on an HCP lattice. The general features of this model are again essentially the same as those of the above models.

The second model we would like to mention here is an example of a theory with the more general type of symmetry of the type (2.21). The theory is described by the hamiltonian

$$
H=\operatorname{Re} \sum_{\boldsymbol{r}}\left[\alpha s(\boldsymbol{r}) s^{\dagger}(\boldsymbol{r}+\hat{z})+\beta s^{\dagger}(\boldsymbol{r}-\hat{\boldsymbol{x}}) s^{\dagger}(\boldsymbol{r}+\tilde{\boldsymbol{x}}) s^{\dagger}(\boldsymbol{r}-\hat{\boldsymbol{y}}) s^{\dagger}(\boldsymbol{r}+\hat{\boldsymbol{y}}) s^{4}(\boldsymbol{r})\right] .
$$

In its periodic gaussian form, the partition function of the model takes the form [4]

$$
Z=\sum_{\{\phi=0\}}^{N-1} \sum_{\{m, n=-\infty\}}^{\infty} \exp \left\{\frac{2 \pi^{2}}{N^{2}} \sum_{J}\left[-\beta\left(\nabla_{p}^{2} \phi_{J}-N n_{j}\right)^{2}-\alpha\left(\nabla_{z} \phi_{J}-N m_{J}\right)^{2}\right]\right\},
$$

where $j$ labels a lattice site and

$$
\begin{aligned}
\nabla_{p}^{2} \phi_{J} & =\phi_{J-\hat{x}}+\phi_{J-\hat{x}}+\phi_{J+\hat{y}}+\phi_{J-\hat{y}}-4 \phi_{J}, \\
\nabla_{z} \phi_{J} & =\phi_{J}-\phi_{J-\hat{z}},
\end{aligned}
$$

where $\hat{x}, \hat{y}, \hat{z}$ represent unit vectors on a cubic lattice. An $n$, is associated with an elementary cross in the $(x, y)$ plane, and an $m_{j}$ is associated with a link in the $z$-direction.

The hamiltonian of this model, considered as a function of the $\phi$ 's (that is, after summing over the $n$ 's and $m$ 's) is invariant under (2.21) with $n=2$ where $O_{1}=\nabla_{p}^{2}$ and $O_{2}=\nabla_{z}$. The phase diagram of this model is similar to that of the models described above, but with two important differences: First, for $N<N_{\mathrm{c}}$, the single transition in this case appears to be second order, rather than first, and for $N \geqslant N_{\mathrm{c}}$, the two transitions are known to be infinite order [4]. Second, the value of $N_{c}$ is not 5, but, as a preliminary Monte Carlo analysis indicates, seems to be close to 11. It is significant that all the known models with symmetry index $n=2$ of the type (2.20) have the same value of $N_{\mathrm{c}}$, while the one that does not fit into this category but rather has a more general symmetry of the type (2.21) has a different value for $N_{\mathrm{c}}$. 


\section{Summary}

In this paper we have presented the results of a first step in a systematic study of theories with unusual symmetries as described in ref. [1]. We have discussed a number of theories with symmetry index $n \leqslant 2$, and have concentrated on three dimensions. In the same sense in which $d=2$ is the lower critical dimension for ordinary spin theories with a continuous global symmetry, $n=2$ is the lower critical value of the symmetry index for our wider class of theories. In our study we found remarkable similarities between the phase diagrams and other properties of our $n=2$ three-dimensional models and globally-symmetric models in two dimensions. We also discussed a model whose $n=2$ symmetry is of a somewhat different character than the $n=2$ symmetries of our other examples. Significantly, the $Z_{N}$ version of this model has a value for $N_{\mathrm{c}}$ which is very different from that shared by all our other models and the $d=2$ clock models. It thus appears that the precise form of the constraints on the gauge function have a strong influence of the value of $N_{c}$.

Our study has left a number of intriguing questions unanswered. In addition to those mentioned in subsect. 2.4 , it would be interesting to know how $N_{c}$ depends on the operators in (2.21) for the case $n=2$. It would also be interesting to know how the critical exponents in our models vary from one to the other, and how they compare with those of the $d=2$ clock models. Series expansion and finite-size scaling techniques might be useful here. Finally, for those theories with $n>2$, it is important to examine the patterns of symmetry breaking that occur. In such theories there are a variety of semi-local symmetries which may or may not be broken at the same point in coupling-constant space as the global symmetry. The study of this important question could uncover new phases and forms of symmetry breaking and will deepen our understanding of critical phenomena in general.

We are happy to acknowledge the hospitality of the Institute for Theoretical Physics at Santa Barbara, where this and related work was begun. The research was supported, in part, by the National Science Foundation under grant PHY77-27084.

\section{Appendix}

Consider the classical hamiltonian in (3.4) and think of the $z$ axis as defining a (euclidean) time direction. In this appendix we will construct an operator, $\hat{H}$, which will describe the evolution of a spin configuration at $z$ into one at $z^{\prime}$. Throughout this appendix, we will label the $z$ direction with an variable, $t$ (with a lattice spacing $\tau$ in the $z$ direction) and consider a cubic lattice of linear dimension $L$. When the spins are $Z_{N}$ variables of the form

$$
s(\boldsymbol{r}, \boldsymbol{t})=\mathrm{e}^{t(2 \pi / N) n(r, t)}, \quad n=0,1, \ldots, n-1,
$$

where $\boldsymbol{r}=(x, y)$ refers only to the spatial coordinates, the system can exist in any 
of $M$ states for each $t$, where

$$
M=N^{L^{2}}
$$

The classical hamiltonian we are interested in is given by

$$
H=\sum_{t}\left[h_{1}(t)+h_{2}(t)\right],
$$

where

$$
\begin{gathered}
h_{2}(t)=\sum_{r}-\frac{1}{2} \alpha_{2}\left[s(r, t) s^{\dagger}(r+\hat{x}, t) s(r+\hat{x}+\hat{y}, t) s^{\dagger}(r+\hat{y}, t)+\text { c.c. }\right], \\
h_{1}(t)=\sum_{r}-\frac{1}{2} \alpha_{1}\left[s(r, t) s^{\dagger}(r, t+\tau)+\text { c.c. }\right]
\end{gathered}
$$

We want to construct a matrix, the transfer matrix, $\hat{T}$, such that the partition function for the model can be written as

$$
Z=\operatorname{Tr}\left[\hat{T}^{L}\right] .
$$

Calling $|n\rangle$ a configuration of the system at time $t$, and $\left|n^{\prime}\right\rangle$ another at time $t^{\prime}$, we can write

$$
\left\langle n^{\prime}|\hat{T}| n\right\rangle=\left\langle n^{\prime}\left|\hat{T}_{1}\right| n\right\rangle\left\langle n\left|\hat{T}_{2}\right| n\right\rangle,
$$

where

$$
\begin{aligned}
\left\langle n\left|\hat{T}_{2}\right| n\right\rangle & =\mathrm{e}^{-\beta h_{2}(t)}, \\
\left\langle n^{\prime}\left|\hat{T}_{1}\right| n\right\rangle & =\mathrm{e}^{-\beta h_{1}(t)},
\end{aligned}
$$

with $h_{1}$ and $h_{2}$ given above. We can think of $s(r, t)$ as the eigenvalue of an operator $S(r, t)$ which acts on the space of configurations,

$$
S(r, t)|n\rangle=\mathrm{e}^{i(2 \pi / N) n(r, t)}|n\rangle .
$$

Because $\hat{T}_{2}$ is diagonal, it can simply be written in terms of $S$. To construct the off-diagonal part of $\hat{T}, \hat{T}_{1}$, notice that, in terms of the integer phase of $s(r, t), n(r, t)$, we can write

$$
\begin{aligned}
\left\langle n^{\prime}\left|\hat{T}_{1}\right| n\right\rangle= & \exp \left(\beta \alpha_{2}\right) \delta_{\mid n-n^{\prime}, 0}+\exp \left[\beta \alpha_{2} \cos \frac{2 \pi}{N}\right] \delta_{\left|n-n^{\prime}\right|, 1} \\
& +\cdots+\exp \left[\beta \alpha_{2} \cos \frac{2 \pi(N-1)}{N}\right] \delta_{\mid n-n^{\prime}, N-1}
\end{aligned}
$$

Because the set of states, $\{|n\rangle\}$, is complete, (A.10) can be written in terms of an operator $R(r)$ which is the $Z_{N}$ conjugate of $S$ :

$$
\left\langle n^{\prime}|R| n\right\rangle=\delta_{n^{\prime}, n+1}(\bmod N) .
$$


It follows that

$$
\begin{gathered}
S(r) R(r)=\mathrm{e}^{\mathrm{t}(2 \pi / N)} R(\boldsymbol{r}) S(r), \\
R^{N}=1,
\end{gathered}
$$

with $\left[S(\boldsymbol{r}), R\left(\boldsymbol{r}^{\prime}\right)\right]=0$ for $\boldsymbol{r} \neq \boldsymbol{r}^{\prime}$. Therefore,

$$
\hat{T}_{1}=\prod_{r} \sum_{k=0}^{N-1} R^{k}(r) \exp \left[\beta \alpha_{1} \cos \frac{2 \pi}{N} k\right],
$$

The operator hamiltonian can now be obtained by considering the limit

$$
\hat{H}=\lim _{\tau \rightarrow 0}\left(-\frac{1}{\tau} \ln \hat{T}\right),
$$

with $\tau$ the spacing in the $t$ direction. The limit must be taken with $\lambda$ fixed, where

$$
\begin{aligned}
& \lambda=\frac{\beta \alpha_{2}}{\tau}, \\
& \tau=\exp \left[\beta \alpha_{1} \cos \frac{2 \pi}{N}\right] .
\end{aligned}
$$

It is easy to see from (A.14) that only the first two terms in the sum contribute, so that we can identify

$$
\hat{H}=-\lambda \sum_{r} \frac{1}{2}\left[S(\boldsymbol{r}) S^{\dagger}(\boldsymbol{r}+\hat{\boldsymbol{x}}) S(\boldsymbol{r}+\hat{\boldsymbol{x}}+\hat{\boldsymbol{y}}) S^{\dagger}(\boldsymbol{r}+\hat{\boldsymbol{y}})+\text { c.c. }\right]-\frac{1}{2} \sum_{\boldsymbol{r}}\left[R(\boldsymbol{r})+R^{\dagger}(\boldsymbol{r})\right] .
$$

The dual operator corresponding to $\hat{H}$ can be obtained quite easily. Define the operators

$$
\begin{gathered}
\mu(\tilde{\boldsymbol{r}})=S(\boldsymbol{r}) S^{\dagger}(\boldsymbol{r}+\hat{\boldsymbol{x}}) S(\boldsymbol{r}+\hat{\boldsymbol{x}}+\hat{\boldsymbol{y}}) S^{\dagger}(\boldsymbol{r}+\hat{\boldsymbol{y}}), \\
\rho(\tilde{\boldsymbol{r}})=\prod_{n_{\mathrm{x}}=0}^{\infty} \prod_{n_{y}=0}^{\infty} R\left(x-n_{x}, y-n_{y}\right),
\end{gathered}
$$

where $\tilde{\boldsymbol{r}}=\boldsymbol{r}+\frac{1}{2}(\hat{\boldsymbol{x}}+\hat{\boldsymbol{y}})$. It is a simple exercise to show that

$$
\rho(\tilde{\boldsymbol{r}}) \mu(\tilde{\boldsymbol{r}})=\mathrm{e}^{i(2 \pi / N)} \mu(\tilde{\boldsymbol{r}}) \rho(\tilde{\boldsymbol{r}})
$$

and that

$$
[\rho(\tilde{\boldsymbol{r}}), \mu(\tilde{\boldsymbol{r}})]=0 .
$$

Furthermore, $\rho^{N}(\tilde{\boldsymbol{r}})=\mu^{N}(\tilde{\boldsymbol{r}})=1$, so the dual operators have the same algebra as the original operators $S$ and $R$. Also,

$$
\rho(\tilde{\boldsymbol{r}}) \rho^{\dagger}(\tilde{\boldsymbol{r}}+\hat{\boldsymbol{x}}) \rho(\tilde{\boldsymbol{r}}+\hat{\boldsymbol{x}}+\hat{\boldsymbol{y}}) \rho^{\dagger}(\tilde{\boldsymbol{r}}+\hat{\boldsymbol{y}})=R(\boldsymbol{x}+\hat{\boldsymbol{x}}+\hat{\boldsymbol{y}})
$$


Therefore,

$$
\begin{aligned}
\hat{H}= & -\lambda\left\{\sum_{\tilde{\boldsymbol{r}}} \frac{1}{2 \lambda}\left[\rho(\tilde{\boldsymbol{r}}) \rho^{\dagger}(\tilde{\boldsymbol{r}}+\hat{\boldsymbol{x}}) \rho(\tilde{\boldsymbol{r}}+\hat{\boldsymbol{x}}+\hat{\boldsymbol{y}}) \rho^{\dagger}(\tilde{\boldsymbol{r}}+\hat{\boldsymbol{y}})+\text { h.c. }\right]\right. \\
& \left.-\frac{1}{2} \sum_{\tilde{\boldsymbol{r}}}\left[\mu(\tilde{\boldsymbol{r}})+\mu^{\dagger}(\tilde{\boldsymbol{r}})\right]\right\} .
\end{aligned}
$$

From (A.17) and (A.23) it follows that

$$
\hat{H}(\lambda)=\lambda \hat{H}(1 / \lambda),
$$

demonstrating the self-duality of the hamiltonian operator. The dual coupling is given by $\tilde{\lambda}=1 / \lambda$, implying that the self-dual point occurs at $\lambda=1$ for all values of $N$.

\section{References}

[1] F.C. Alcaraz, L Jacobs and R. Savit, Nucl Phys B257 [FS14] (1985) 340

[2] F C. Alcaraz, L. Jacobs and R. Savit, Phys Rev. Lett 51 (1983) 435

[3] S Elitzur, R Pearson and J. Shigemitsu, Phys Rev. D19 (1979) 3698;

A. Ukawa, P. Windey and A Guth, Phys. Rev D21 (1980) 1013;

E. Fradkin and L Kadanoff, Nucl. Phys B170 [FS1] (1980) 1;

M. Einhorn, E. Rabınovicı and R Savit, Nucl Phys. B170 [FS1] (1980) 16,

L. Kadanoff, J. Phys A11 (1978) 1399

[4] D Amit, S. Elitzur, E Rabınov1c1 and R. Savit, Nucl Phys B210 [FS6] (1982) 69

[5] F.C Alcaraz, L Jacobs and R Savit, J Phys. A16 (1982) 175

[6] F C. Alcaraz, L. Jacobs and R. Savit, Phys. Lett 89A (1982) 49 ,

R Liebmann, Phys. Lett 85A (1981) 59, Z. Phys. B45 (1982) 243;

P. Pearce and R. Baxter, Phys. Rev. B24 (1981) 5295;

L Jacobs and R Savit, Ann. of the New York Academy of Sciences, 410 (1983) 281

[7] N.D. Mermin and H. Wagner, Phys Rev. Lett. 17 (1966) 1133;

F. Wegner, Z. Phys. 206 (1967) 465,

N D. Mermın, Phys Rev. 176 (1968) 250

[8] N N Bogoliubov, Physik Abhandl Sowjetunion 6 (1962) 1, 113, 229

H. Wagner, Z. Phys. 195 (1966) 273

[9] S Elitzur, Phys Rev D12 (1975) 3978

[10] F.C. Alcaraz and L. Jacobs, J. Phys A15 (1982) L357

[11] R Savit, Rev. Mod Phys. 52 (1980) 453

[12] R. Savit, Nucl Phys B200 [FS4] (1982) 233

[13] See, e.g., M Creutz, L Jacobs and C. Rebbi, Phys Reports 95 (1983) 201

[14] M Creutz, L Jacobs and C. Rebb1, Phys Rev D20 (1979) 1915 